\title{
Billroth II gastrectomy complicated by gastro- jejunocolonic fistulas, treated endoscopically with a cardiac septal defect closure device
}

Gastrojejunocolonic fistula is a severe complication of gastrectomy with mainly Billroth II reconstruction carried out for peptic ulcer or malignant disease. It may develop 1-20 years after the procedure. Since the small bowel is bypassed, malnutrition due to malabsorption occurs [1,2]. A 58-year-old Greek man was admitted for fecal-smelling eructation, diarrhea, and weight loss during the past year. He had undergone a distal gastric resection with Billroth II reconstruction for a perforated duodenal ulcer 21 years ago. Barium meal and computed tomography enteroclysis studies revealed fistulous tracts between the transverse colon, the upper jejunum, and the gastric remnant ( $\bullet$ Fig. 1). Upper gastrointestinal endoscopy confirmed the above findings and showed fecal material into the gastric remnant lumen ( Fig. 2). The patient refused surgery and after malignancy was excluded, we attempted to occlude both fistulas with the Amplatzer atrial septal defect closure device (9-ASD-040; AGA Medical Corp., Plymouth, Minnesota, USA), an idea based on the report of Melmed et al. [3]. We modified the technique of device delivery by using the endoscope itself to upload, guide, and deploy the Amplatzer device through the wide fistula tract $(\bullet$ Fig. 3, $\bullet$ Video 1 ).

The procedure was uneventful and 1 week later, an endoscopy showed the device at the gastroenterostomy site without fecal material into the gastric lumen ( $\bullet$ Fig. 4); a small but functionally insignificant leakage of Gastrografin was also noted ( $\bullet$ Fig. 5). The patient's condition improved with cessation of fecal-smelling eructation and diarrhea and an increase in appetite and weight.

It is recommended that malnutrition should be corrected and radical surgery carried out with resection of the entire

\section{Video 1}

Technique for endoscopic implantation of an Amplatzer device for the occlusion of a gastrocolonic fistula.

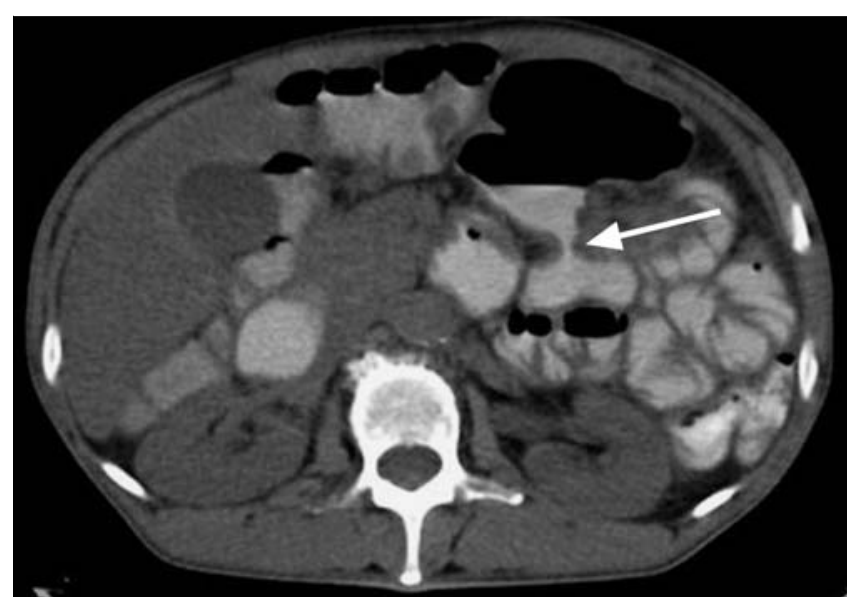

Fig. 1 Axial computed tomography $(\mathrm{CT})$ enteroclysis demonstrating a fistula between the greater curvature of the stomach and the transverse colon (arrow).

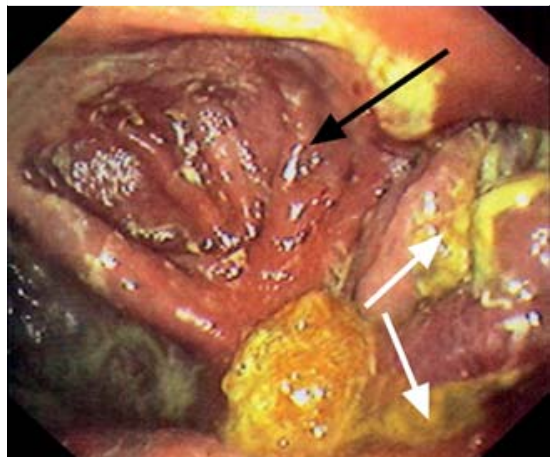

Fig. 2 Endoscopic appearance of two neighboring fistulas discharging fecal material (white arrows) close to the gastroenteroanastomosis (black arrow).

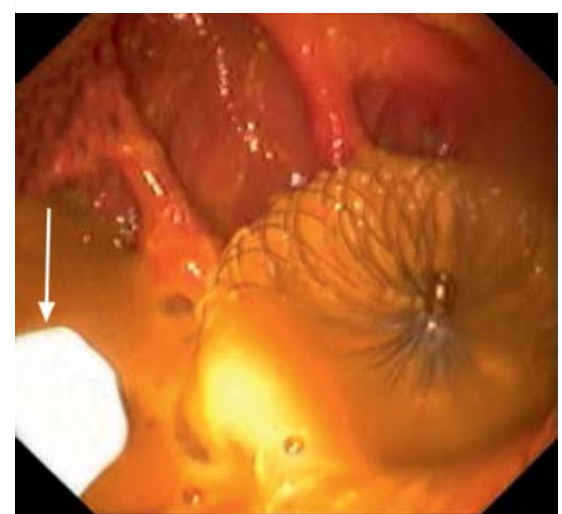

Fig. 3 Endoscopic view of the orifices of the two gastrojejunocolic fistulas occluded by the Amplatzer device. The arrow is indicating the tip of the delivery catheter attached to the endoscope.

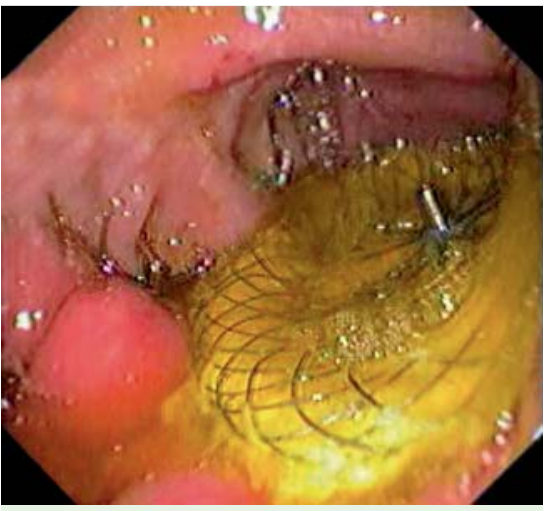

Fig. 4 Endoscopic appearance of the bilestained Amplatzer device 1 week after placement.

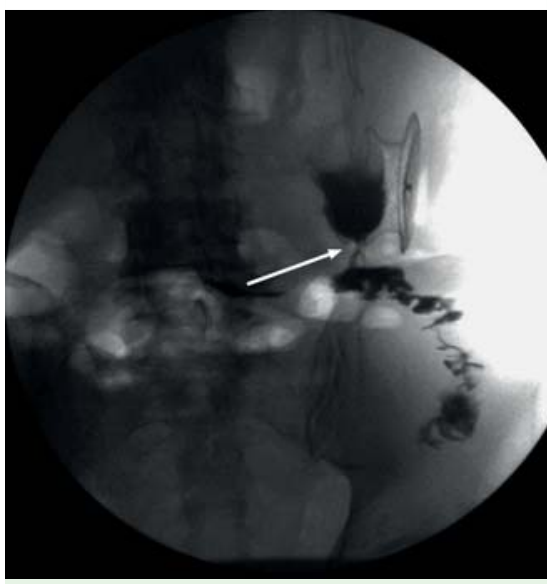

Fig. 5 Upper gastrointestinal study with Gastrografin 1 week after the placement of the Amplatzer device. 
fistula and re-establishment of gastrojejunal and colonic continuity $[2,4]$. However, nonoperative medical management strategies have also been proposed [5]. This is the first case of implantation of an Amplatzer atrial septal defect closure device to occlude two gastrojejunocolonic fistulas with a novel delivery method in the complicated setting of a Billroth II reconstruction. This approach could be an alternative to surgical management in certain circumstances, especially in patients with a high operative risk.

\section{Competing interests: None}

Endoscopy_UCTN_Code_TTT_1AO_2AI

\section{G. Kouklakis ${ }^{1}$, P. Zezos ${ }^{1}$, N. Liratzopou- $\operatorname{los}^{2}$, M. Pitiakoudis ${ }^{3}$, E. Efremidou ${ }^{2}$, A. Giatromanolaki ${ }^{4}$, N. Courcoutsakis ${ }^{5}$, C. Simopoulos ${ }^{3}$}

1 Gastrointestinal Endoscopy Unit, Democritus University of Thrace, University General Hospital of Alexandroupolis, Alexandroupolis, Greece
2 1st Department of Surgery, Democritus University of Thrace, University General Hospital of Alexandroupolis, Alexandroupolis, Greece

3 2nd Department of Surgery, Democritus University of Thrace, University General Hospital of Alexandroupolis, Alexandroupolis, Greece

4 Department of Pathology, Democritus University of Thrace, University General Hospital of Alexandroupolis, Alexandroupolis, Greece

${ }^{5}$ Department of Radiology, Democritus University of Thrace, University General Hospital of Alexandroupolis, Alexandroupolis, Greece

\section{References}

1 Cody JH, DiVincenti FC, Cowick DR et al. Gastrocolic and gastrojejunocolic fistulae: report of twelve cases and review of the literature. Ann Surg 1975; 181: 376 - 380

2 Ohta M, Konno H, Tanaka T et al. Gastrojejunocolic fistula after gastrectomy with Billroth II reconstruction: report of a case. Surg Today 2002; 32: 367-370

3 Melmed GY, Kar S, Geft I et al. A new method for endoscopic closure of gastrocolonic fis- tula: novel application of a cardiac septal defect closure device (with video). Gastrointest Endosc 2009; 70: 542 - 545

4 Filipovic B, Randjelovic T, Nikolić G. Gastrojejunocolic fistula as a complication of Billroth II gastrectomy: a case report. Acta Chir Belg 2008; 108: 592 - 594

5 Rots WI, Mokoena T. Successful endoscopic closure of a benign gastrocolonic fistula using human fibrin sealant through gastroscopic approach: a case report and review of the literature. Eur J Gastroenterol Hepatol 2003; 15: $1351-1356$

\section{Bibliography}

DOI $10.1055 / \mathrm{s}-0029-1244058$

Endoscopy 2010; 42: E134-E135

(c) Georg Thieme Verlag KG Stuttgart · New York . ISSN 0013-726X

\section{Corresponding author}

\section{P. Zezos, MD}

Gastrointestinal Endoscopy Unit, Democritus University of Thrace, University General Hospital of Alexandroupolis 40 Venizelou Str

68100 Alexandroupolis

Greece

Fax: +30-25510-84168

zezosp@hol.gr 